\title{
Thrombospondin-1 Exacerbates Acute Liver Failure and Hepatic Encephalopathy Pathology in Mice by Activating Transforming Growth Factor $\beta 1$
}

Brandi Jefferson, ${ }^{*}$ Malaika Ali, ${ }^{*}$ Stephanie Grant, ${ }^{\dagger \ddagger}$ Gabriel Frampton, ${ }^{\dagger \S}$ Michaela Ploof, ${ }^{*}$ Sarah Andry, ${ }^{\llbracket}$ Sharon DeMorrow, ${ }^{* \dagger \dagger \S}$ and Matthew McMillin*§

From the Central Texas Veterans Health Care System, * Austin; the Department of Medical Physiology, ${ }^{\dagger}$ Texas A\&M University Health Science Center, Temple; the Division of Pharmacology and Toxicology, ${ }^{\ddagger}$ College of Pharmacy, The University of Texas at Austin, Austin; the Department of Internal Medicine, ${ }^{\S}$ The University of Texas at Austin Dell Medical School, Austin; and the Department of Internal Medicine, ${ }^{\Uparrow}$ Baylor Scott \& White Health, Temple, Texas

Accepted for publication October 21, 2019.

Address correspondence to Matthew McMillin, Ph.D., Department of Internal Medicine, University of Texas at Austin Dell Medical School, 1601 Trinity St., Bldg. B, Austin, TX 78701. E-mail: matthew. momillin@austin.utexas.edu.

\begin{abstract}
Severe hepatic insults can lead to acute liver failure and hepatic encephalopathy (HE). Transforming growth factor $\beta 1$ (TGF $\beta 1$ ) has been shown to contribute to HE during acute liver failure; however, TGF $\beta 1$ must be activated to bind its receptor and generate downstream effects. One protein that can activate TGF $\beta 1$ is thrombospondin-1 (TSP-1). Therefore, the aim of this study was to assess TSP-1 during acute liver failure and $\mathrm{HE}$ pathogenesis. $\mathrm{C} 57 \mathrm{Bl} / 6$ or TSP-1 knockout $\left(\mathrm{TSP}-1^{-/-}\right)$mice were injected with azoxymethane (AOM) to induce acute liver failure and HE. Liver damage, neurologic decline, and molecular analyses of TSP-1 and TGF $\beta 1$ signaling were performed. AOM-treated mice had increased TSP1 and TGF $\beta 1$ mRNA and protein expression in the liver. TSP $-1^{-/}$mice administered AOM had reduced liver injury as assessed by histology and serum transaminase levels compared with $\mathrm{C} 57 \mathrm{Bl} / 6 \mathrm{AOM}$-treated mice. TSP $-1^{-1-}$ mice treated with AOM had reduced TGF $\beta 1$ signaling that was associated with less hepatic cell death as assessed by terminal deoxynucleotidyl transferase-mediated dUTP nick-end labeling staining and cleaved caspase 3 expression. TSP-1 ${ }^{-/-}$AOM-treated mice had a reduced rate of neurologic decline, less cerebral edema, and a decrease in microglia activation in comparison with C57BL/6 mice treated with AOM. Taken together, TSP-1 is an activator of TGF $\beta 1$ signaling during AOMinduced acute liver failure and contributes to both liver pathology and HE progression. (Am J Pathol 2020, 190: 347-357; https://doi.org/10.1016/j.ajpath.2019.10.003)
\end{abstract}

Acute liver failure results from a significant loss of liver function caused by hepatocyte necrosis in response to hepatotoxin ingestion, viral hepatitis, autoimmune disease, metabolic disease, ischemia, and various other causes. Patients with acute liver failure often present with deleterious systemic complications, including neurologic dysfunction called hepatic encephalopathy (HE). ${ }^{1} \mathrm{HE}$ is a serious neuropsychiatric complication that is responsible for approximately $20 \%$ to $25 \%$ of deaths resulting from acute liver failure and negatively influences health-related quality of life, clinical management strategies, liver transplant priority, and survival. ${ }^{1,2} \mathrm{HE}$ resulting from acute liver failure generates changes in mental status, including cognitive disruptions that can progress to hepatic coma in hours or days resulting from the development of cerebral edema and increased intracranial pressure.,

Aberrant cellular signaling pathways can contribute to the pathogenesis of acute liver failure and HE. One of these

\footnotetext{
Supported by US Department of Veterans Affairs Biomedical Laboratory Research and Development Service VA Career Development award BX003486 (M.M.), NIH R01 awards DK082435 and DK112803, US Department of Veterans Affairs Biomedical Laboratory Research and Development Service VA Merit award BX002638 (S.D.), and the Central Texas Veterans Health Care System (Temple, TX).

The content is the responsibility of the author(s) alone and does not necessarily reflect the views or policies of the Department of Veterans Affairs or the United States Government.

Disclosures: None declared.
} 
pathways is transforming growth factor beta 1 (TGF 31 ). Plasma concentrations of transforming growth factor $\beta 1$ (TGF $\beta 1$ ) are increased in patients with acute liver failure. ${ }^{5}$ In mice with acute liver failure, TGF $\beta 1$ has been shown to be up-regulated and colocalizes with markers of hepatocytes. ${ }^{6,7}$ The use of a pan-TGF $\beta$ inhibitor in mice with acute liver failure slows the progression of HE. ${ }^{6}$ In addition, hepatic TGF $\beta 1$ enters the brain by permeabilizing the blood-brain barrier, subsequently inducing TGF $\beta$ receptor $2-$ mediated signaling in neurons, leading to increased neuroinflammation in the cerebral cortex. ${ }^{7,8}$

TGF $\beta 1$ is a member of a multifunctional cytokine family that binds and activates a heterotetramer-receptor complex made up of TGF $\beta$ receptors 1 and 2, leading to phosphorylation of SMAD2 and SMAD3 proteins, which ultimately modulate transcription of numerous genes. ${ }^{9,10}$ TGF $\beta 1$ is secreted in its inactive form associated with latencyassociated peptide, and must be released from this complex to be active and bind its receptors. ${ }^{11}$ One of the proteins responsible for the activation of TGF $\beta 1$ is thrombospondin-1 (TSP-1), which can bind the latent complex at the conserved sequence leucine-serine-lysine-leucine (LSKL) on the latency-associated peptide, resulting in the release of TGF $\beta 1$ from the latent complex. ${ }^{12}$

TSP-1 is a matricellular glycoprotein that was first isolated and characterized from human blood platelets. ${ }^{13}$ This protein has numerous functions including regulating angiogenesis, apoptosis, inflammation, cell fate determination, extracellular matrix deposition, and other cellular functions. ${ }^{14,15}$ These various functions are the result of the numerous ligands that interact with TSP-1, with interactome studies identifying 83 different targets. ${ }^{16}$ Although TSP-1 has not been studied extensively during acute liver failure, TSP-1 has been shown to influence both lipid accumulation in nonalcoholic fatty liver disease and the inhibition of liver regeneration by activating latent TGF $\beta 1 .^{17,18}$

Currently, little data exist concerning the involvement of TSP-1 in TGF $\beta 1$ activation during acute liver failure and the development of HE. Our aim was to assess TSP-1 expression and how it influences the activation state and downstream signaling of TGF $\beta 1$ during acute liver failure and HE.

\section{Materials and Methods}

\section{Materials}

All chemicals used were of the highest necessary grade and were purchased from Millipore-Sigma (Burlington, MA) unless noted otherwise. RNeasy mini kits and real-time PCR primers against TSP-1 (catalog number PPM03098F-200), TGF $\beta 1$ (catalog number PPM02991B-200), chemokine ligand 2 (catalog number PPM03151G-200), and glyceraldehyde 3phosphate dehydrogenase (catalog number PPM02946E-200) were purchased from Qiagen (Germantown, MD). The iScript cDNA kit, Laemmli buffer, running buffer, and transfer buffer were purchased from Bio-Rad (Hercules, CA). Hematoxylin $\mathrm{QS}$, antigen unmasking solution, and VectaStain $\mathrm{ABC}$ kits were purchased from Vector Laboratories (Burlingame, CA). TGF $\beta 1$ and TSP-1 antibodies were purchased from Santa Cruz Biotechnology (Dallas, TX). The cleaved caspase 3 antibody was purchased from Cell Signaling Technology (Danvers, MA). Ionized calcium-binding adapter molecule 1 (IBA1) antibodies were purchased from Wako Chemicals USA (Richmond, VA). Blocking buffer and secondary antibodies for Western blot were ordered from Li-Cor Biosciences (Lincoln, NE). Terminal deoxynucleotidyl transferasemediated dUTP nick-end labeling (TUNEL) kits were purchased from Abcam (Cambridge, MA). Fluorescent secondary antibodies for immunofluorescence were bought from Jackson ImmunoResearch Laboratories (West Grove, PA).

\section{Azoxymethane Model of Acute Liver Failure}

TSP-1 null mice $\left(\mathrm{TSP}^{-1}{ }^{-/}\right.$, stock 006141; B6.129S2Thbs $\left.1^{\mathrm{tm} 1 \mathrm{Hyn}} / \mathrm{J}\right)$ or $\mathrm{C} 57 \mathrm{Bl} / 6 \mathrm{~J}$ mice were from The Jackson Laboratory (Bar Harbor, ME) and used for all in vivo experiments. Acute liver failure and $\mathrm{HE}$ were caused by a single intraperitoneal injection of $100 \mathrm{mg} / \mathrm{kg}$ body weight of azoxymethane (AOM) into mice (20 to $25 \mathrm{~g}$ ) as described previously. ${ }^{19-22}$ After AOM administration, mice cages were placed on heating pads set to $37^{\circ} \mathrm{C}$ to ensure the mice remained normothermic. Hydrogel and rodent chow were placed on the floor of the cages to ensure access to food and hydration. Mice were injected subcutaneously with $5 \%$ dextrose in $250 \mu \mathrm{L}$ saline at 12 hours and every 4 hours thereafter to ensure euglycemia and hydration. Mice were monitored every 2 hours (starting at 12 hours after AOM injection) for body temperature, weight, and neurologic score using previously published methodology. ${ }^{6,23,24}$ After the development of neurologic dysfunction, mice were monitored with formal assessments of temperature, body weight, and neurologic score performed every hour until the mice became comatose. The neurologic score was determined by an investigator blind to the treatments (S.G. or G.F.) by assigning a score between 0 (absent) and 2 (intact) to the following parameters: the pinna reflex, corneal reflex, tail flexion, escape response, righting reflex, and ataxia. The scores of these five reflexes and ataxia were summated to provide a neurologic score between 0 and 12 . Once mice lost their corneal and righting reflex, the time to coma was recorded, the mice were euthanized, and tissue was collected to be used for all analyses.

\section{Cerebral Edema}

Cerebral edema was assessed in all mice using the wet/dry weight method as previously described. ${ }^{25}$ This method involves weighing a micro-centrifuge tube before and after placing brain tissue inside of it to calculate the weight of the tube and the wet weight of the tissue, respectively. The microcentrifuge tube is left uncapped and put into an oven at $70^{\circ} \mathrm{C}$ for 2 days. The microcentrifuge tube with the tissue is weighed again to calculate the dry weight. Water content was expressed as a percentage of brain weight; calculated as 
follows: ((wet weight - dry weight)/wet weight $) \times 100 \%$. An increase in brain water content of $1 \%$ to $2 \%$ in mice is indicative of cerebral edema and increased intracranial pressure. ${ }^{26,27}$

\section{Liver Histology and Serum Chemistry}

Paraffin-embedded livers were cut into $4-\mu \mathrm{m}$ sections and mounted onto positively charged slides (VWR, Radnor, PA). Slides were deparaffinized with xylene and rehydrated with ethanol at decreasing concentrations. The liver tissue then was stained with Hematoxylin QS (Vector Laboratories) and Eosin Y (Amresco, Solon, $\mathrm{OH}$ ) and rinsed with 95\% ethanol. The slides were submerged into $100 \%$ ethanol and then through two xylene washes. Coverslips were mounted onto the slides using CytoSeal XYL mounting media (ThermoFisher Scientific, Waltham, MA). The slides were imaged using an Olympus BX40 microscope with a DP25 imaging system (Olympus, Center Valley, PA).

Liver function was assessed by measuring serum alanine aminotransferase levels using a Catalyst One serum chemistry analyzer from IDEXX Laboratories, Inc. (Westbrook, MA).

\section{mRNA Analysis}

Liver and cortex tissue from vehicle, AOM, and TSP-1 ${ }^{-1-}$ mice were homogenized and RNA was isolated using an RNeasy Mini Kit (Qiagen) according to the manufacturer's instructions. The concentration of RNA in each sample was measured using a ThermoFisher Scientific Nanodrop 2000 spectrophotometer. cDNA was synthesized using a Bio-Rad iScript cDNA Synthesis Kit and real-time PCR was performed as described previously ${ }^{28}$ using commercially available primers designed against mouse TSP-1, TGF $\beta 1$, $C c l 2$, and $G A P D H$. SYBR green fluorescence was measured using a MX3005P thermal cycler from Agilent Technologies (Santa Clara, CA). A $\Delta \Delta \mathrm{Ct}$ analysis was performed using vehicle-treated tissue as the control group. ${ }^{29,30}$

\section{TUNEL Assay and Immunohistochemistry}

TUNEL assays and immunohistochemistry were performed in liver sections (4- to 6- $\mu \mathrm{m}$ thick) prepared as outlined in Liver Histology and Serum Chemistry. The TUNEL assays were performed according to the manufacturer's protocols with no modifications. For immunohistochemistry, antibodies against TSP-1, TGF $\beta 1$, phosphorylated mothers against decapentaplegic homolog 3 (pSMAD3), and cleaved caspase 3 were incubated overnight at $4^{\circ} \mathrm{C}$. Subsequent incubation with secondary antibody and color development using 3,3'-diaminobenzidine substrate was performed using Vector Laboratories kits according to the manufacturer's instructions. Sections were counterstained with Hematoxylin QS. TUNEL and immunohistochemical stained tissue were scanned by a Leica SCN400 digital slide scanner (Leica Microsystems, Buffalo Grove, IL). The percentage area staining positive for pSMAD3 or TUNEL staining was quantified by converting images to grayscale and quantifying positive staining area using ImageJ software version 1.52a (NIH, Bethesda, MD; https://imagej.nih.gov/ij).

\section{Western Blot}

Liver and cortex tissue from each mouse group were homogenized using a Miltenyi Biotec (Auburn, CA) gentleMACS Dissociator and total protein concentrations were measured using a ThermoFisher BCA Protein Assay kit. SDSPAGE gels (10\% to $15 \% \mathrm{v} / \mathrm{v})$ were loaded with 30 to $40 \mu \mathrm{g}$ protein diluted in Laemmli buffer per tissue sample. TSP-1, TGF $\beta 1$, cytochrome $\mathrm{p} 4502 \mathrm{E} 1$, cleaved caspase 3 , and $\beta$-actin antibodies were used. Imaging was performed on a Li-Cor Odyssey 9120 Infrared Imaging System. Data are expressed as fold change in fluorescent band intensity of the target antibody compared with $\beta$-actin, which was used as a loading control. The control group values were used as a baseline and set to a relative protein expression value of 1 . Band intensity quantifications were performed using ImageJ software.

\section{Immunofluorescence}

Free-floating immunofluorescence staining was performed on 30- $\mu \mathrm{m}$ thick brain sections. Brain sections were blocked with $5 \%$ goat serum and then were incubated with IBA1 antibodies to detect morphology and relative staining of microglia. Cy3 fluorescent secondary antibodies were used to visualize immunoreactivity. Brain sections subsequently were moved to positively charged slides and coverslips were mounted using ProLong Gold Antifade Reagent (ThermoFisher) containing DAPI. Brain sections were imaged using a Leica TCS SP5-X inverted confocal microscope (Leica Microsystems). The field fluorescence area of IBA1 was calculated by converting images to grayscale, inverting their color, and quantifying field staining with ImageJ software.

\section{Statistical Analysis}

Statistical analyses were performed using GraphPad Prism 8 version 8.2.1 (GraphPad Software, La Jolla, CA). Results were expressed as means \pm SEM. Significance was determined using the $t$-test when differences between two groups were assessed, and analysis of variance when differences between three or more groups were compared. A two-way analysis of variance was performed for the neurologic score analyses followed by a Bonferroni multiple comparison post hoc test. Differences were considered significant for $P$ values $<0.05$.

\section{Results}

\section{TSP-1 Expression Increases during AOM-Induced HE}

Mice were administered AOM to induce acute liver failure and HE. After AOM treatment, there was a significant increase of hepatic TSP-1 mRNA expression compared with 

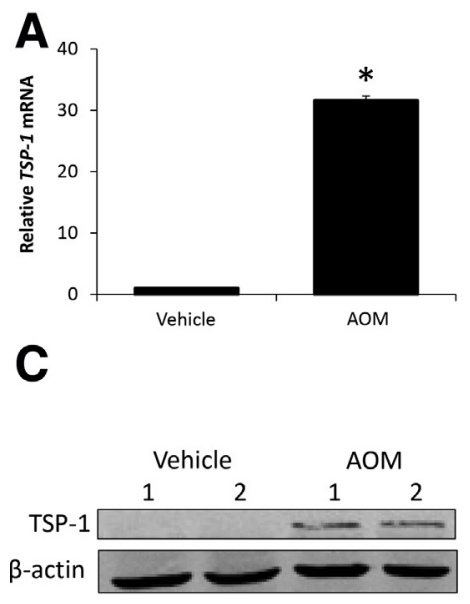

B

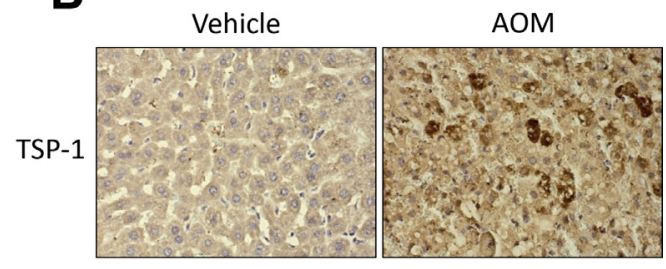

D

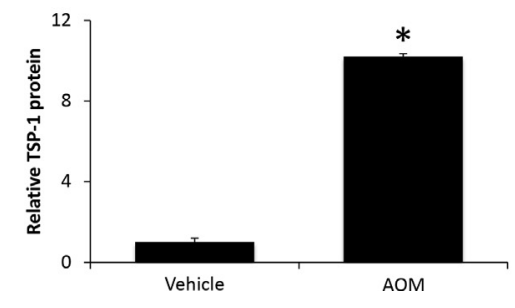

Figure 1 Thrombospondin-1 (TSP-1) expression is increased during azoxymethane (AOM)induced acute liver failure. A: Relative TSP-1 mRNA expression in liver homogenates from vehicle- and AOM-treated mice. B: Immunohistochemical staining images for TSP-1 in liver sections from vehicle- and AOM-treated mice. C: Immunoblot images for TSP-1 in liver homogenates from vehicle- and AOM-treated mice. $\beta$-actin was used as a protein loading control. D: Relative immunoblot TSP-1 expression normalized to $\beta$-actin in the livers from vehicle- and AOM-treated mice. $n=4$ liver homogenates from vehicle- and AOM-treated mice (A and $\mathbf{D}) .{ }^{*} P<0.05$ versus vehicle-treated mice. Original magnification, $\times 400$. vehicle-treated mice (Figure 1A). In liver sections from AOM-treated mice, staining for TSP-1 was increased compared with vehicle-treated mice, with distinct hepatocyte populations near areas of necrosis expressing high TSP-1 levels (Figure 1B). TSP-1 protein expression in the liver was increased significantly in AOM-treated mice, with levels nearly undetectable in vehicle-treated mice (Figure 1, C and D). Collectively, these data support an increase of hepatic TSP-1 expression in response to AOM-induced acute liver injury.

\section{A0M Treatment Increased TGF $\beta 1$ Expression}

Because TSP-1 can increase TGF $\beta 1$ activity and subsequent signaling, the expression of TGF $\beta 1$ was assessed in vehicle and AOM-treated mice. AOM-induced acute liver failure generated an increase of TGF $\beta 1$ mRNA expression in the liver compared with vehicle-treated mice (Figure 2A). In liver sections from AOM-treated mice, there was increased staining for TGF $\beta 1$ compared with sections from vehicletreated mice (Figure 2B). In support of these data, TGF $\beta 1$ protein expression was increased significantly in liver homogenates from AOM-treated mice compared with vehicletreated mice (Figure 2, C and D). SMAD3 phosphorylation, as a measure of downstream TGF $\beta 1$ activity, was increased significantly in the livers of AOM-treated mice, however, staining was not observed in vehicle-treated livers (Figure 2, $\mathrm{E}$ and $\mathrm{F}$ ).

\section{TSP-1 $1^{-/-}$Mice Were Protected from AOM-Induced Liver} Injury

Because of the correlation between TSP-1 expression and increased TGF $\beta 1$ expression and activity in the livers of AOM-treated mice, the effects of genetic ablation of TSP-1 were examined during AOM-induced HE. Vehicle-treated wild-type (WT) or TSP- $1^{-1-}$ mice administered AOM had low TSP-1 expression in the liver, whereas hepatic TSP-1 expression was increased significantly in WT AOMtreated mice (Figure 3, A and B). To validate that TSP-1 exacerbated AOM-induced hepatotoxicity, liver injury and function were assessed. In WT AOM-treated mice there was extensive necrosis, microvesicular steatosis, and hemorrhage present, which were reduced in $\mathrm{TSP}_{-} 1^{-1-}$ mice injected with AOM (Figure 3C). These histologic findings are mirrored by alanine aminotransferase levels, which show a large increase in WT AOM-treated mice that was reduced significantly in $\mathrm{TSP}^{-1} 1^{-1}$ mice administered $\mathrm{AOM}$ (Figure 3D). Because it is possible that the hepatoprotective effects of TSP- $1^{-1-}$ compared with WT mice were the result of differences in AOM metabolism, cytochrome p450 2E1 expression was assessed. Cytochrome p450 2E1 was reduced significantly in both WT AOM-treated and TSP- $1^{-1-}$ AOM-treated mice, with no significant differences between groups (Figure 3, E and F).

\section{TGF $\beta 1$ Activation and Cell Death Was Decreased in $\mathrm{TSP}^{-1}{ }^{-/-}$Mice}

With TGF $\beta 1$ being known to contribute to AOM pathogenesis, the hypothesis that TSP- 1 activates TGF $\beta 1$ during AOM-induced liver injury was evaluated. TGF $\beta 1$ mRNA expression was increased significantly in WT AOM-treated mice, but was reduced significantly in $\mathrm{TSP}^{-1} 1^{-1}$ mice treated with AOM (Figure 4A). TGF $\beta 1$ staining in liver sections was observed throughout WT AOM-treated mice whereas TSP- $1^{-1-}$ mice treated with AOM showed reduced staining, to levels near WT vehicle-treated mice (Figure 4B). These staining results were validated by immunoblot data, showing a significant increase of active TGF $\beta 1$ in WT AOM-treated mice, which was reduced significantly in $\mathrm{TSP}_{-1} 1^{-1-}$ mice injected with AOM (Figure 4, C and D). Staining for pSMAD3 was widespread in liver sections from WT AOM-treated mice and the area of staining was reduced significantly in $\mathrm{TSP}^{-1} 1^{-1-} \mathrm{AOM}-$ treated mice (Figure 4, E and F). 
A

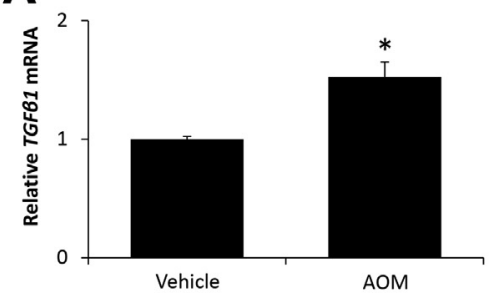

C

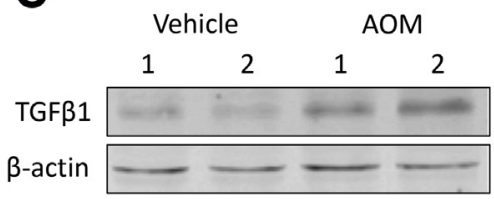

E

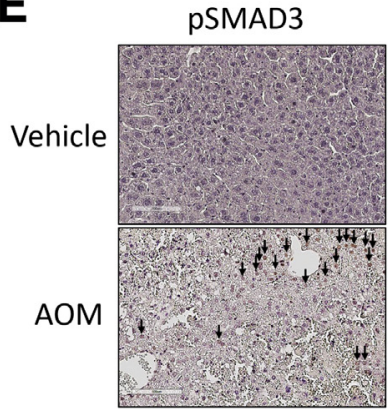

B

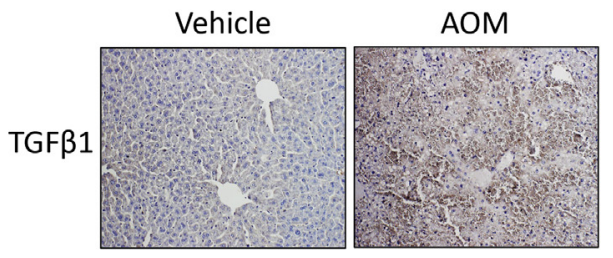

D

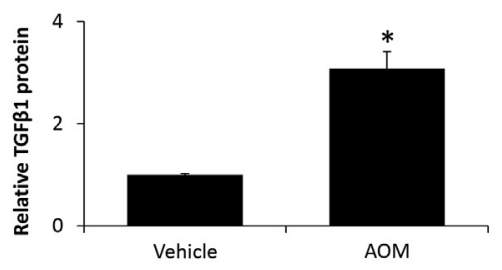

$\mathbf{F}$

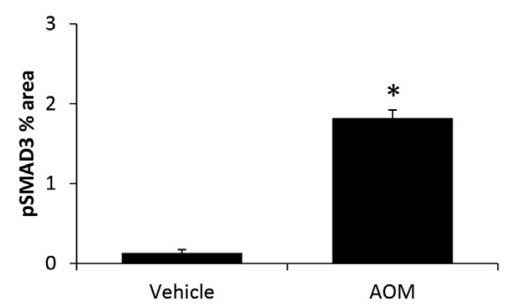

Figure 2 Transforming growth factor $\beta 1$ (TGFB1) signaling is activated during azoxymethane (AOM)-induced acute liver failure. $\mathbf{A}$ : Relative TGF $\beta 1$ mRNA expression in liver homogenates from vehicle- and AOM-treated mice. B: Immunohistochemical staining images for TGF $\beta 1$ in liver sections from vehicle- and AOM-treated mice. C: Immunoblot images for TGF $\beta 1$ in liver homogenates from vehicle- and AOM-treated mice. $\beta$-actin was used as a protein loading control. D: Relative immunoblot TGF $\beta 1$ expression normalized to $\beta$-actin in livers from vehicle- and $\mathrm{AOM}$-treated mice. $\mathbf{E}$ and $\mathbf{F}$ : Immunohistochemical staining images and quantification of the percentage area stained for phosphorylated SMAD3 (pSMAD3) in liver sections from vehicle- and AOM-treated mice. pSMAD3-positive staining is indicated with arrows. $n=4$ liver homogenates from vehicle- and A0M-treated mice (A and $\mathbf{D}-\mathbf{F})$. ${ }^{*} P<0.05$ versus vehicle-treated mice. Original magnification, $\times 200($ B and E).
TGF $\beta 1$ can be an inducer of apoptosis, which could drive AOM-induced liver injury. TUNEL staining was increased significantly in WT AOM-treated mice in areas neighboring necrotic tissue, although there was a significant reduction in TUNEL-positive cells in $\mathrm{TSP}-1^{-1-}$ AOM-treated mice (Figure 5, A and B). In addition, cleaved caspase 3 staining was increased in WT
AOM-treated mice and significantly reduced in TSP- $1^{-1-}$ AOM-treated mice, although a small degree of staining still was observed (Figure 5C). Immunoblots for cleaved caspase 3 show similar results, with a significant increase of cleaved caspase 3 expression in WT AOM-treated mice, which was reduced significantly in $\mathrm{TSP}-1^{-1}$ AOM-treated mice (Figure 5, D and E).
A

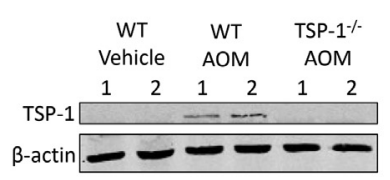

D

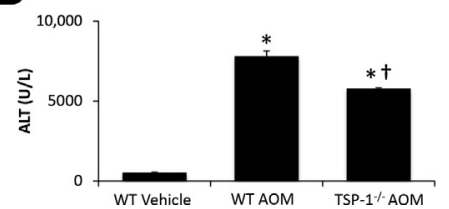

B

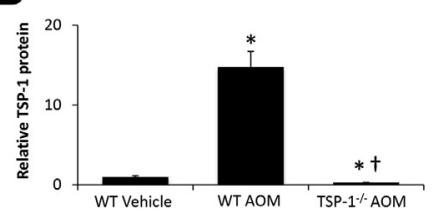

$\mathbf{E}$

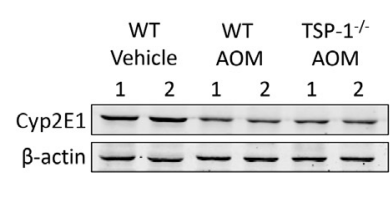

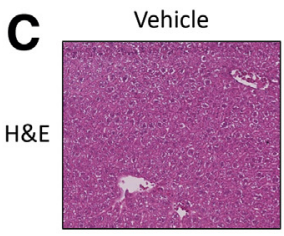

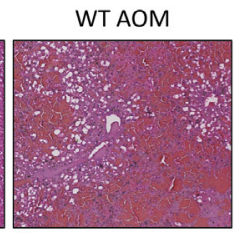

TSP- $1 \%$ AOM
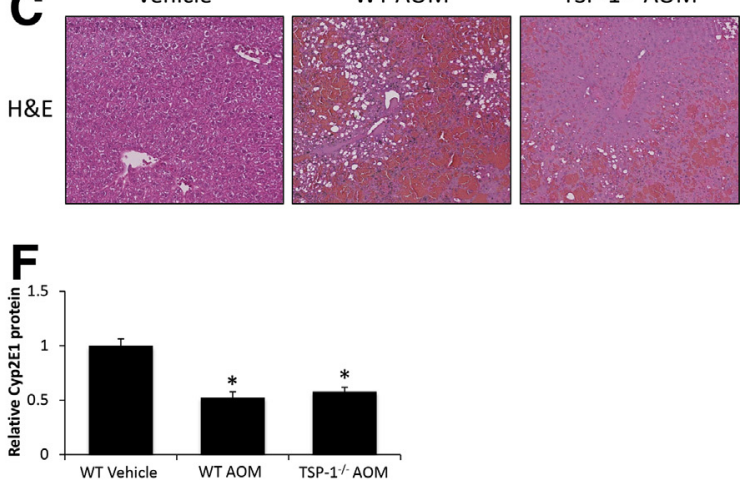

Figure $3 \mathrm{TSP}-1^{-1-}$ mice were protected from azoxymethane (AOM)-induced liver injury. A: Immunoblot images for thrombospondin-1 (TSP-1) in liver homogenates from wild-type (WT) or TSP-1 ${ }^{-/}$mice treated with vehicle or AOM. $\beta$-actin was used as a protein loading control. B: Relative immunoblot TSP-1 expression was normalized to $\beta$-actin in livers from WT or TSP-1 $1^{-/-}$mice administered vehicle or AOM. C: Hematoxylin and eosin (H\&E) staining images in liver sections from WT or TSP-1 $1^{-/-}$mice treated with vehicle or AOM. D: Serum alanine aminotransferase (ALT) concentrations in WT or TSP-1 ${ }^{-1-}$ mice treated with vehicle or A0M. E: Immunoblot images for cytochrome p450 2E1 (Cyp2E1) in liver homogenates from WT or TSP- $1^{-/-}$mice administered vehicle or A0M. $\beta$ actin was used as a protein loading control. F: Relative immunoblot Cyp2E1 expression normalized to $\beta$-actin in livers from WT or TSP-1 ${ }^{-/-}$mice treated with vehicle or AOM. $n=4$ livers from WT or TSP- $1^{-/-}$mice administered vehicle or AOM (A and F); $n=5$ WT or TSP- $1^{-1-}$ mice treated with vehicle or A0M (D). ${ }^{*} P<0.05$ versus $W T$ vehicle-treated mice; ${ }^{\dagger} P<0.05$ versus WT AOM-treated mice. Original magnification, $\times 200$. 

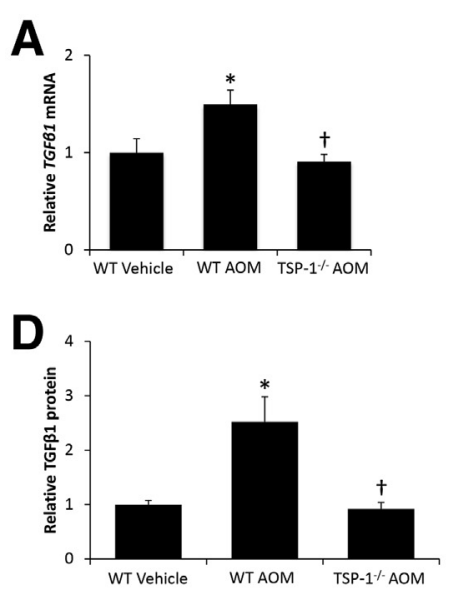

B
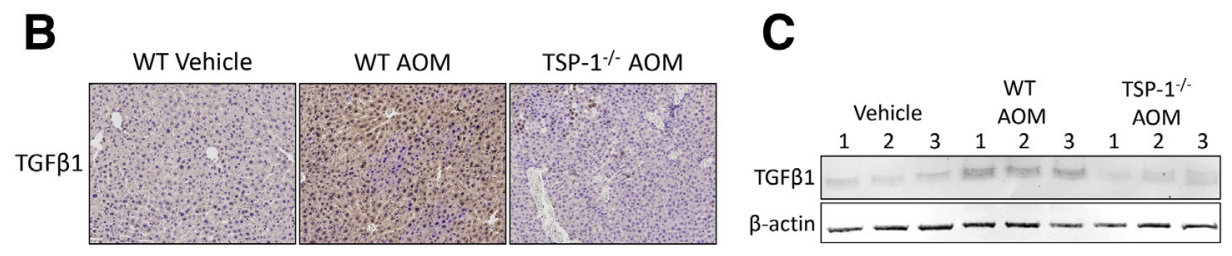

$\mathbf{E}$

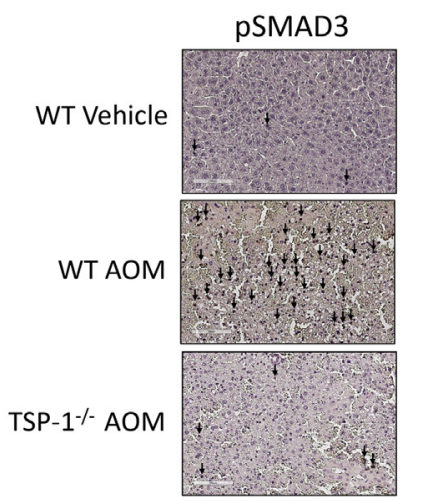

$\mathbf{F}$

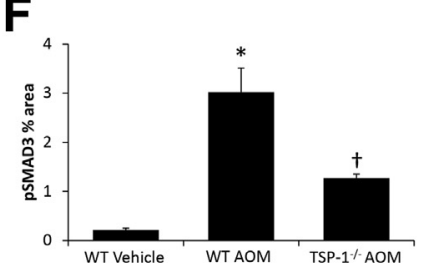

Figure 4 Hepatic transforming growth factor $\beta 1$ (TGF $\beta 1$ ) activation is reduced in thrombospondin-1 null (TSP-1 ${ }^{-/}{ }^{-}$) mice. A: Relative TGF $\beta 1$ mRNA expression in livers from wild-type (WT) or TSP-1 $1^{-1-}$ mice administered vehicle or AOM. B: Immunohistochemical staining images for TGF $\beta 1$ in liver sections from WT or TSP-1 $1^{-1-}$ mice treated with vehicle or AOM. C: Immunoblot images for TGF $\beta 1$ in liver homogenates from WT or TSP- $1^{-/-}$mice treated with vehicle or AOM. $\beta$-actin was used as a protein loading control. D: Relative immunoblot TGF $\beta 1$ expression normalized to $\beta$-actin in livers from WT or TSP-1 ${ }^{-/-}$mice administered vehicle or AOM. E and F: Immunohistochemical staining images and the percentage area staining quantification for phosphorylated SMAD3 (pSMAD3) in liver sections from WT or TSP-1 ${ }^{-1-}$ mice treated with vehicle or AOM. Cells with pSMAD3-positive staining are marked with arrows. $n=4$ livers from wild-type (WT) or TSP- $1^{-1-}$ mice administered vehicle or AOM (A and $\left.\mathbf{D}\right)$ and liver sections from WT or TSP- $1^{-1-}$ mice treated with vehicle or A0M (E and F). ${ }^{*} P<0.05$ versus WT vehicle-treated mice; ${ }^{\dagger} P<0.05$ versus WT A0M-treated mice. Original magnification, $\times 200(\mathbf{B}$ and $\mathbf{E})$.

\section{Improved Neurologic Outcomes Were Observed in TSP $-1^{-/-}$Mice}

The rate of neurologic decline was measured in the WT and TSP- $1^{-1-}$ mice after the injection of AOM. The mice were assigned a neurologic score ranging between 0 and 12 , with 12 indicating full neurologic capabilities and 0 indicating an absence of reflexes and the onset of hepatic coma. TSP-1 ${ }^{-/-}$ knockout mice injected with AOM had a delayed rate of neurologic decline compared with WT AOM-treated mice (Figure 6A). TSP-1 ${ }^{-1-}$ AOM-treated mice had a significantly increased latency to reach coma in comparison with the AOM-injected WT mice (Figure 6B). Cerebral edema was increased significantly in WT AOM-treated mice and reduced significantly in $\mathrm{TSP}-1^{-1-}$ AOM-treated mice, to levels similar to WT vehicle-treated mice (Figure 6C).

\section{Microglia Activation Was Reduced in A0M-Treated TSP-1 ${ }^{-/-}$Mice}

Microglia in the brain are activated during HE and, as a result, experiments were conducted to assess microglial activation after AOM injection. Immunofluorescence was conducted using the microglia marker IBA1, which showed that WT AOM-treated mice have increased field staining for IBA1 that is reduced in TSP-1 ${ }^{-1-}$ AOM-treated mice (Figure 7, A and $\mathrm{B}$ ). In addition, the morphology of microglia in WT AOM-treated mice show more of an amoeboid-type shape with shorter processes, an indication of activation
(Figure 7A). This change in morphology was not observed in the $\mathrm{TSP}-1^{-1-}$ AOM-treated mice, which have microglia similar to WT vehicle-treated mice (Figure 7A). Microglia activation during $\mathrm{HE}$ can be a result of increased chemokine ligand 2 expression, ${ }^{31,32}$ and $C c l 2$ mRNA levels were found to be up-regulated significantly in WT AOM-treated mice, with a significant decrease in TSP- $1^{-1-}$ AOM-treated mice (Figure 7C).

\section{Discussion}

The data presented in the current study support that AOMinduced acute liver injury leads to increased TSP-1 expression in the liver and increased pSMAD3 signaling as a result of increased levels of activated TGF $\beta 1$ in the liver. During AOM-induced acute liver injury, TSP-1 expression was increased throughout the liver, with hepatocytes showing the highest expression levels. Eliminating the up-regulation of TSP-1 through the use of TSP- $1^{-/-}$mice showed that TSP-1 induced TGF $\beta 1$ activation and signaling, exacerbated liver injury, promoted hepatic cell death, and, ultimately, led to worse liver function when these mice were compared with control mice. In addition, TSP- $1^{-1-}$ mice administered AOM have a reduced rate of neurologic decline and less microglia activation when compared with WT controls. Taken together, these data support that increased TSP-1 activity contributes to the pathogenesis of acute liver failure and HE.

Although the role of TSP-1 during acute liver failure has not been investigated directly, there is evidence that TSP-1 

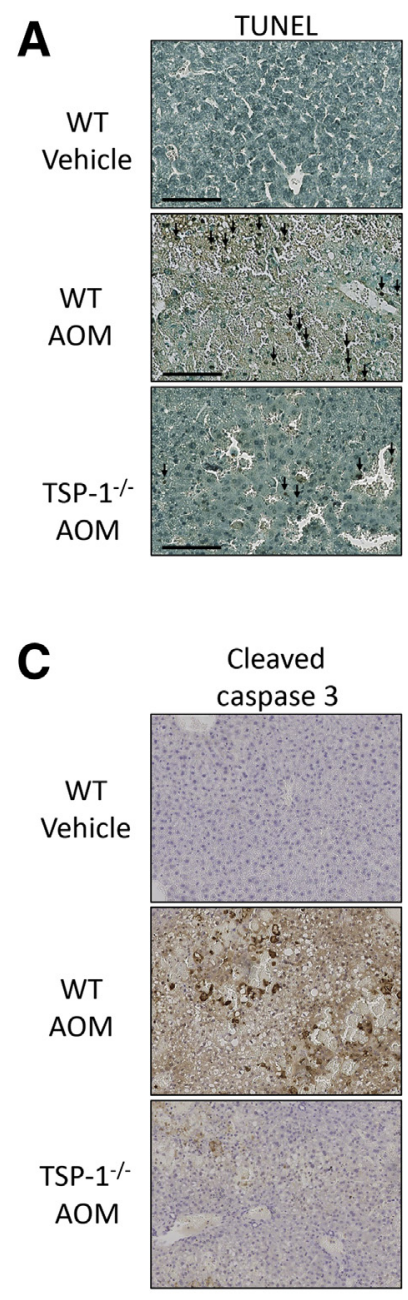

B

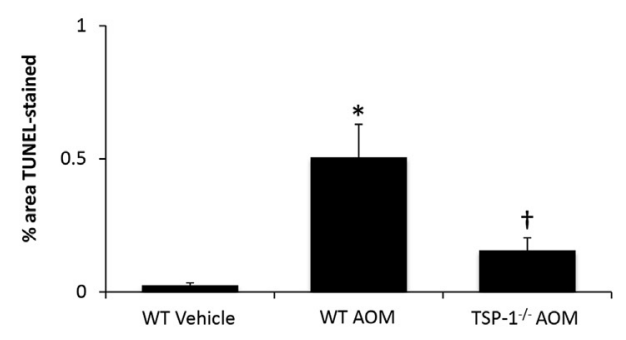

D

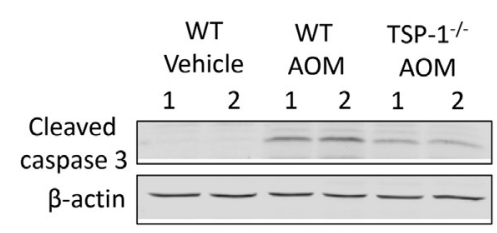

E

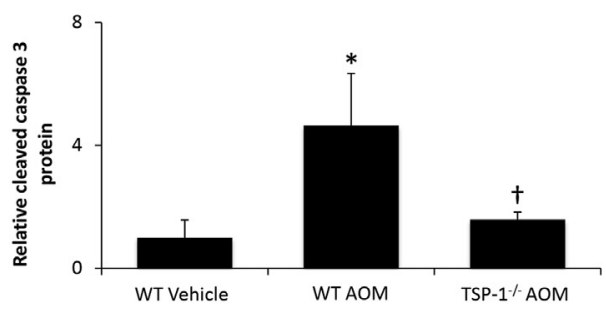

Figure 5 Hepatic cell death is reduced in thrombospondin-1 null (TSP-1 ${ }^{-/}$) mice. A: Terminal deoxynucleotidyl transferase-mediated dUTP nick-end labeling (TUNEL) staining images in liver sections from wild-type (WT) or $\mathrm{TSP}-1^{-/-}$mice administered vehicle or azoxymethane (AOM). TUNEL-positive staining is indicated with black arrows. B: Quantification of TUNEL-positive area per field in liver sections from WT or TSP-1 ${ }^{-1-}$ mice treated with vehicle or AOM. C: Immunohistochemical staining images for cleaved caspase 3 in liver sections from WT or TSP-1 ${ }^{-/-}$mice administered vehicle or AOM. D: Immunoblot images for cleaved caspase 3 in liver homogenates from WT or $\mathrm{TSP}-1^{-/-}$mice treated with vehicle or AOM. $\beta$ actin was used as a protein loading control. E: Relative cleaved caspase 3 expression as assessed with immunoblots in liver homogenates from WT or TSP $-1^{-/-}$mice administered vehicle or AOM. $n=$ 3 liver sections from WT or TSP-1 ${ }^{-1-}$ mice treated with vehicle or AOM (B); $n=4$ liver homogenates from WT or TSP-1/mice administered vehicle or AOM (E). ${ }^{*} P<0.05$ versus WT vehicle-treated mice; ${ }^{\dagger} P<0.05$ versus wild-type A0M-treated mice. Scale bars $=100 \mu \mathrm{m}(\mathbf{A})$. Original magnification, $\times 200(C)$. is associated with acute liver injury. In patients undergoing liver resection, levels of circulating TSP-1 were increased significantly at 1 day after surgery, but not at 5 days after surgery, and platelet counts did not increase 1 day after resection, indicating that platelets were not the source of TSP-1. ${ }^{33}$ In the same study, the investigators divided the patients into TSP-1 low-expression and TSP-1 highexpression groups and found that the group with higher TSP-1 levels took longer to recover liver function after liver resection, as assessed by alanine aminotransferase level, aspartate aminotransferase level, bilirubin level, and prothrombin time. ${ }^{33}$ The effect of TSP-1 on slowing the rate of recovery likely is owing to a reduced rate of liver regeneration. This is supported by rodent studies that showed administration of the TSP-1 inhibitory peptide leucine-serine-lysine-leucine to mice with $70 \%$ hepatectomy led to increased cell proliferation 24 hours after surgery. ${ }^{34} \mathrm{~A}$ study by Hayashi et $\mathrm{al}^{18}$ found a similar result by
A

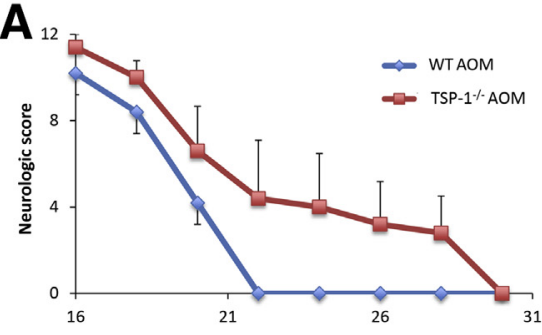

B

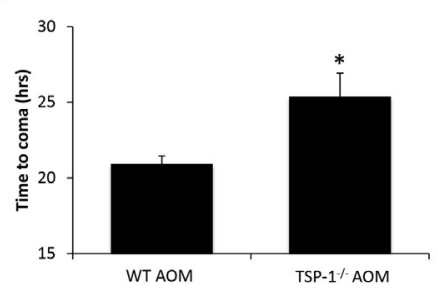

C

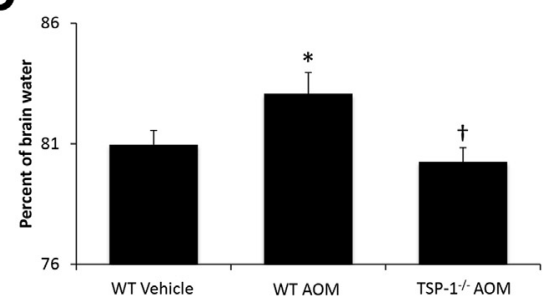

Figure 6 AOM-induced neurologic decline is reduced in thrombospondin-1 null (TSP-1 ${ }^{-/}$) mice. A: Neurologic score in wild-type (WT) and TSP-1 ${ }^{-/-}$ azoxymethane (AOM)-treated mice on a scale from 0 to 12, with a higher score indicating better neurologic function. B: Time to progress to coma measured in hours in WT and TSP-1 $1^{-1-}$ mice. C: Brain water percentage in WT or TSP- $1^{-1-}$ mice treated with vehicle or AOM. $n=5$ WT and TSP-1 $1^{-1-}$ mice (B) and WT and $\mathrm{TSP} 1^{-/-}$mice treated with vehicle or AOM (C). ${ }^{*} P<0.05$ versus WT vehicle-treated mice; ${ }^{\dagger} P<0.05$ versus WT A0M-treated mice. 


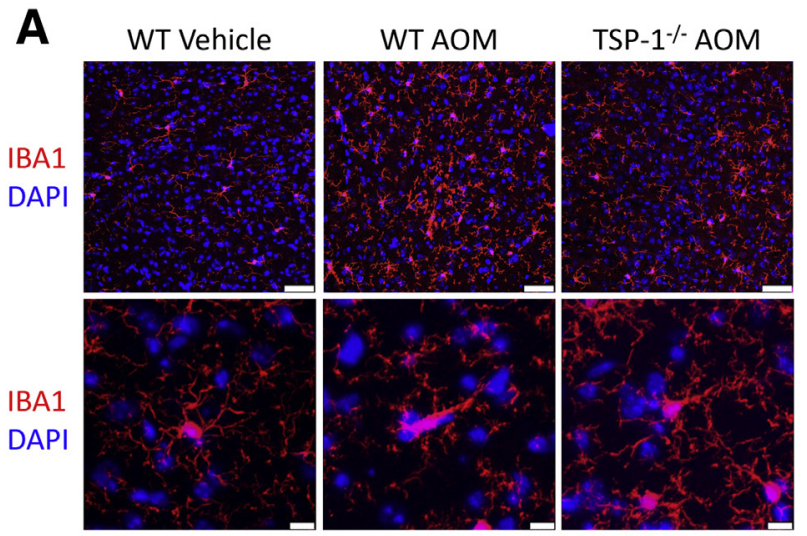

\section{B}

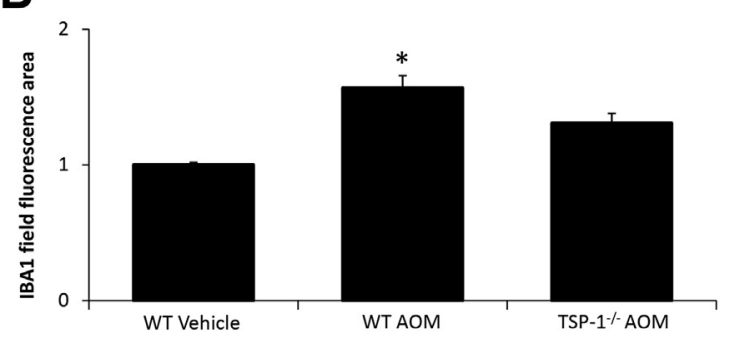

C

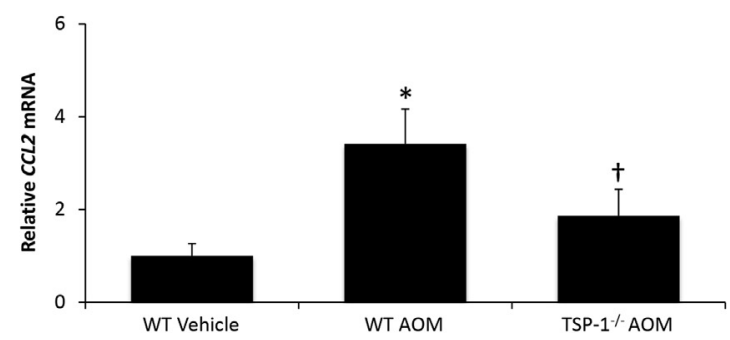

Figure 7 Microglia activation and neuroinflammation are reduced in thrombospondin-1 null (TSP-1 ${ }^{-/}$) azoxymethane (AOM)-treated mice. A: Immunofluorescence images for ionized calcium-binding adapter molecule 1 (IBA1) staining (red) to visualize microglia in field views (top row) and Z-stack images (bottom row), with DAPI (blue) used to stain nuclei in the cortex of wild-type (WT) or TSP-1 ${ }^{-1-}$ mice administered vehicle or AOM. B: Quantification of relative field IBA1 staining in the cortex of WT or TSP-1 ${ }^{-1-}$ mice treated with vehicle or AOM. C: Relative cortical Ccl2 mRNA expression in WT or TSP-1 $1^{-1-}$ mice treated with vehicle or AOM. $n=4$ WT or TSP-1 ${ }^{-1-}$ mice treated with vehicle or AOM (B and $\mathbf{C}$ ). ${ }^{*} P<0.05$ versus WT vehicle-treated mice; ${ }^{\dagger} P<0.05$ versus WT AOM-treated mice. Scale bars: $50 \mu \mathrm{m}$ (A, top row); $10 \mu \mathrm{m}$ (A, bottom row).

performing $70 \%$ hepatectomy in $\mathrm{TSP}^{-1-}{ }^{-1}$ mice and observed increased proliferation at 24 hours after surgery. In the current study, TSP-1 was up-regulated in the liver, with specific hepatocyte populations showing the highest expression levels of this protein. It is possible that this increase of expression occurs before the hepatocytes undergo apoptosis, owing to TSP-1 being shown to induce apoptosis through a p38 mitogen-activated protein kinase-dependent mechanism. ${ }^{35}$ AOM-induced liver injury was reduced in TSP- $1^{-1-}$ mice, supporting a role for this signaling pathway in acute liver injury. It should be noted that TSP-1 has additional functions aside from activating TGF $\beta 1$, and can interact with $\mathrm{CD} 36, \mathrm{CD} 47$, and other proteins, indicating that some of the observations regarding TSP-1 in this study could be a result of interactions with proteins outside of TGF $\beta 1$, which is an area under current investigation ${ }^{36,37}$

In the context of the current study, TGF $\beta 1$ has been shown to inhibit liver regeneration, and injection of TGF $\beta 1$ into rats at the time of partial hepatectomy and 11 hours later significantly reduced hepatic DNA synthesis measured 22 hours after surgery. ${ }^{38}$ In addition, inhibition of TGF $\beta$ receptor type 1 with LY364947 in mice treated with carbon tetrachloride increased proliferating cell nuclear antigen expression and reduced the expression of cyclin-dependent kinase inhibitor $\mathrm{p} 21 .{ }^{39}$ Besides inhibiting cell proliferation, TGF $\beta 1$ signaling can induce cell death, leading to overall increased liver injury. The use of LY364947 in mice with carbon tetrachloride-induced liver injury reduced the number of TUNEL-positive cells in the liver compared with carbon tetrachloride-treated control mice. ${ }^{39}$ The use of antibodies against TGF $\beta 1$ in TSP-1-treated cells reduced apoptosis, indicating that the TSP-1-dependent activation of TGF $\beta 1$ is contributing to apoptosis. ${ }^{35}$ In the current study, increased TGF $\beta 1$ activity also was observed and inhibition of TSP-1 activity led to reduced levels of active TGF $\beta 1$ and reduced pSMAD3 signaling. This provides support to the hypothesis that even though TGF $\beta 1$ has the capability to be activated by other proteins, it is likely the large increase of TSP-1 observed during AOM-induced acute liver failure that leads to the majority of TGF $\beta 1$ activation during this disease state. This observation is supported by the decrease of TGF $\beta 1$ signaling, but also by the reduction of TUNEL- and cleaved-caspase 3-stained hepatocytes, indicating a reduction of apoptotic pathways after inhibition of TSP-1. It is worth noting that TSP-1 signaling in itself has been linked to apoptosis, so it is possible that some of the hepatic effects observed in this study were independent of TGF $\beta 1$ signaling.

The neurologic dysfunction that results from the development of HE can be a consequence of astrocyte swelling and cerebral edema in response to increased ammonia levels. ${ }^{40}$ In cirrhotic patients with overt HE, white matter and the thalamus had higher water content when compared with cirrhotic patients without HE or healthy controls. ${ }^{41}$ Diffusion tensor imaging in patients with acute hepatic failure showed that both cytotoxic and vasogenic edema were present in this patient population. ${ }^{42}$ That being said, TSP-1 has not been studied extensively in the context of edema or blood-brain barrier function. One study showed that during traumatic brain injury in mice, TSP-1 is upregulated in the brain and TSP-1 null mice had worse blood-brain barrier leakage. ${ }^{43}$ This does not align with the results of the current study, in which WT mice administered AOM had increased cerebral edema that was not present in TSP- $1^{-1-}$ mice administered AOM. This likely was due to the liver being the site of injury during AOM-induced 
hepatotoxicity and that TSP-1 activated hepatic TGF $\beta 1$ in this model. TGF $\beta 1$ has been shown to induce blood-brain barrier opening during AOM-induced HE through the down-regulation of claudin-5 and the up-regulation of matrix metalloproteinase- $9 .{ }^{8}$ Therefore, it is likely that the activation of hepatic TGF $\beta 1$ by TSP-1 during AOMinduced liver injury lead to the increase of brain water content found in this study. That being said, directly delineating the independent effects of TSP-1 and TGF $\beta 1$ in the context of blood-brain barrier dysfunction and cerebral edema are research areas that warrant further investigation.

Neuroinflammation is a significant contributor to the pathogenesis of HE because microglia activation has been observed in both animal models and patients with acute liver failure. ${ }^{2}$ Microglia are able to be activated by chemokines through the disruption of the balance between proinflammatory chemokines, such as chemokine ligand 2, and anti-inflammatory chemokines, such as CX3CL1. ${ }^{44}$ This applies directly to inflammation during $\mathrm{HE}$ because antagonism of chemokine ligand 2-mediated signaling or increasing CX3CL1 signaling slows the rate of neurologic decline in AOM-treated mice. ${ }^{24,45}$ The current study observed that increased microglia activation and $\mathrm{Ccl} 2$ mRNA expression were associated with AOM-induced neurologic decline. TSP- $1^{-1-}$ mice had reduced measures of neuroinflammation compared with WT controls, indicating an involvement of TSP-1 in this aspect of pathology. These effects are to be expected as a result of the reduced activation of TGF $\beta 1$. Injection of pan-TGF $\beta$ or TGF $\beta 1$ antibodies to neutralize TGF $\beta 1$ signaling have been shown to increase survival of AOM-treated mice. ${ }^{6,7}$ In addition, specific knockout of TGF $\beta$ receptor 2 in neurons increased the time taken to reach coma, reduced microglia activation, and decreased the levels of pro-inflammatory cytokine and chemokine expression. ${ }^{7}$ Therefore, it is likely that the inhibition of TGF $\beta 1$ activity, rather than the direct effects of TSP-1 inhibition in the brain, generated the effects observed in this model. This is supported by a study in which rats administered thioacetamide for 10 days to generate HE had decreased levels of TSP-1 in astrocytes and this correlated with decreased neuronal synaptophysin levels, which could explain impaired neuronal function. ${ }^{46}$

The development of HE pathology after liver injury or loss of function can be the result of many factors including an increase of ammonia, cerebral edema, neuroinflammation, increase of bile acids in the brain, and various other pathologic mediators. ${ }^{47-50}$ Clinical treatments for HE that commonly are used are lactulose and rifaximin, which reduce ammonia levels and have been shown to improve outcomes. $^{51,52}$ That being said, these treatments have differing degrees of therapeutic efficacy depending on the metric assessed. Therefore, the need to develop new therapies, or therapies to use in conjunction with current treatments, for management of this syndrome is critically important. ${ }^{53}$ This study showed that genetic ablation of TSP-1 led to a reduced rate of neurologic decline in the
AOM mouse model of acute liver failure and HE. Therefore, it is possible that targeting TSP-1 signaling may reduce mortality and morbidity in HE patients. However, it will be necessary to investigate this pathway in other preclinical studies before determining its viability as a treatment strategy for this patient population.

In conclusion, the results of this study suggest that after acute liver failure there is up-regulation of TSP-1 in the liver, which results in increased TGF $\beta 1$ activation. Elimination of TSP- 1 through the use of TSP- $1^{-1-}$ mice identified that TSP-1 activates TGF $\beta 1$ in the liver, resulting in worse neurologic outcomes owing to increased neuroinflammation. Therefore, treatments aimed at decreasing TSP-1 concentrations may be a potential therapeutic strategy for the management of HE.

\section{References}

1. Bernal W, Auzinger G, Dhawan A, Wendon J: Acute liver failure. Lancet 2010, 376:190-201

2. Butterworth RF: Hepatic encephalopathy: a central neuroinflammatory disorder? Hepatology 2011, 53:1372-1376

3. Hazell AS, Butterworth RF: Hepatic encephalopathy: an update of pathophysiologic mechanisms. Proc Soc Exp Biol Med 1999, 222: 99-112

4. Fridman V, Galetta SL, Pruitt AA, Levine JM: MRI findings associated with acute liver failure. Neurology 2009, 72:2130-2131

5. Miwa Y, Harrison PM, Farzaneh F, Langley PG, Williams R, Hughes RD: Plasma levels and hepatic mRNA expression of transforming growth factor-betal in patients with fulminant hepatic failure. J Hepatol 1997, 27:780-788

6. McMillin M, Galindo C, Pae HY, Frampton G, Di Patre PL, Quinn M, Whittington E, DeMorrow S: Gli1 activation and protection against hepatic encephalopathy is suppressed by circulating transforming growth factor beta1 in mice. J Hepatol 2014, 61:1260-1266

7. McMillin M, Grant S, Frampton G, Petrescu AD, Williams E, Jefferson B, Thomas A, Brahmaroutu A, DeMorrow S: Elevated circulating TGFbeta1 during acute liver failure activates TGFbetaR2 on cortical neurons and exacerbates neuroinflammation and hepatic encephalopathy in mice. J Neuroinflammation 2019, 16:69

8. McMillin MA, Frampton GA, Seiwell AP, Patel NS, Jacobs AN, DeMorrow S: TGFbetal exacerbates blood-brain barrier permeability in a mouse model of hepatic encephalopathy via upregulation of MMP9 and downregulation of claudin-5. Lab Invest 2015, 95: 903-913

9. Abdollah S, Macias-Silva M, Tsukazaki T, Hayashi H, Attisano L, Wrana JL: TbetaRI phosphorylation of Smad2 on Ser465 and Ser467 is required for Smad2-Smad4 complex formation and signaling. J Biol Chem 1997, 272:27678-27685

10. Zhang Y, Feng X, We R, Derynck R: Receptor-associated Mad homologues synergize as effectors of the TGF-beta response. Nature 1996, 383:168-172

11. Munger JS, Harpel JG, Gleizes PE, Mazzieri R, Nunes I, Rifkin DB: Latent transforming growth factor-beta: structural features and mechanisms of activation. Kidney Int 1997, 51:1376-1382

12. Ribeiro SM, Poczatek M, Schultz-Cherry S, Villain M, MurphyUllrich JE: The activation sequence of thrombospondin-1 interacts with the latency-associated peptide to regulate activation of latent transforming growth factor-beta. J Biol Chem 1999, 274: 13586-13593

13. Lawler JW, Slayter HS, Coligan JE: Isolation and characterization of a high molecular weight glycoprotein from human blood platelets. J Biol Chem 1978, 253:8609-8616 
14. Mirochnik Y, Kwiatek A, Volpert OV: Thrombospondin and apoptosis: molecular mechanisms and use for design of complementation treatments. Curr Drug Targets 2008, 9:851-862

15. Lopez-Dee Z, Pidcock K, Gutierrez LS: Thrombospondin-1: multiple paths to inflammation. Mediators Inflamm 2011, 2011:296069

16. Resovi A, Pinessi D, Chiorino G, Taraboletti G: Current understanding of the thrombospondin-1 interactome. Matrix Biol 2014, 37: $83-91$

17. Li Y, Turpin CP, Wang S: Role of thrombospondin 1 in liver diseases. Hepatol Res 2017, 47:186-193

18. Hayashi H, Sakai K, Baba H, Sakai T: Thrombospondin-1 is a novel negative regulator of liver regeneration after partial hepatectomy through transforming growth factor-betal activation in mice. Hepatology 2012, 55:1562-1573

19. Silva VR, Secolin R, Vemuganti R, Lopes-Cendes I, Hazell AS: Acute liver failure is associated with altered cerebral expression profiles of long non-coding RNAs. Neurosci Lett 2017, 656:58-64

20. McMillin M, Frampton G, Grant S, Khan S, Diocares J, Petrescu A, Wyatt A, Kain J, Jefferson B, DeMorrow S: Bile acid-mediated sphingosine-1-phosphate receptor 2 signaling promotes neuroinflammation during hepatic encephalopathy in mice. Front Cell Neurosci 2017, 11:191

21. Popek M, Bobula B, Sowa J, Hess G, Polowy R, Filipkowski RK, Frontczak-Baniewicz M, Zablocka B, Albrecht J, Zielinska M: Cortical synaptic transmission and plasticity in acute liver failure are decreased by presynaptic events. Mol Neurobiol 2018, 55:1244-1258

22. Chastre A, Belanger M, Nguyen BN, Butterworth RF: Lipopolysaccharide precipitates hepatic encephalopathy and increases blood-brain barrier permeability in mice with acute liver failure. Liver Int 2014, 34:353-361

23. McMillin M, Frampton G, Tobin R, Dusio G, Smith J, Shin H, Newell-Rogers K, Grant S, DeMorrow S: TGR5 signaling reduces neuroinflammation during hepatic encephalopathy. J Neurochem 2015, 135:565-576

24. McMillin M, Frampton G, Thompson M, Galindo C, Standeford H, Whittington E, Alpini G, DeMorrow S: Neuronal CCL2 is upregulated during hepatic encephalopathy and contributes to microglia activation and neurological decline. J Neuroinflammation 2014, 11:121

25. Baskaya MK, Dogan A, Rao AM, Dempsey RJ: Neuroprotective effects of citicoline on brain edema and blood-brain barrier breakdown after traumatic brain injury. J Neurosurg 2000, 92:448-452

26. Cao C, Yu X, Liao Z, Zhu N, Huo H, Wang M, Ji G, She H, Luo Z, Yue S: Hypertonic saline reduces lipopolysaccharide-induced mouse brain edema through inhibiting aquaporin 4 expression. Crit Care 2012, 16:R186

27. Bemeur C, Vaquero J, Desjardins P, Butterworth RF: N-acetylcysteine attenuates cerebral complications of non-acetaminophen-induced acute liver failure in mice: antioxidant and anti-inflammatory mechanisms. Metab Brain Dis 2010, 25:241-249

28. Frampton G, Invernizzi P, Bernuzzi F, Pae HY, Quinn M, Horvat D, Galindo C, Huang L, McMillin M, Cooper B, Rimassa L, DeMorrow S: Interleukin-6-driven progranulin expression increases cholangiocarcinoma growth by an Akt-dependent mechanism. Gut 2012, 61:268-277

29. DeMorrow S, Francis H, Gaudio E, Venter J, Franchitto A, Kopriva S, Onori P, Mancinelli R, Frampton G, Coufal M, Mitchell B, Vaculin B, Alpini G: The endocannabinoid anandamide inhibits cholangiocarcinoma growth via activation of the noncanonical Wnt signaling pathway. Am J Physiol Gastrointest Liver Physiol 2008, 295:G1150-G1158

30. Livak KJ, Schmittgen TD: Analysis of relative gene expression data using real-time quantitative PCR and the 2(-Delta Delta $\mathrm{C}(\mathrm{T})$ ) method. Methods 2001, 25:402-408

31. Dhanda S, Gupta S, Halder A, Sunkaria A, Sandhir R: Systemic inflammation without gliosis mediates cognitive deficits through impaired BDNF expression in bile duct ligation model of hepatic encephalopathy. Brain Behav Immun 2018, 70:214-232
32. Zhang L, Tan J, Jiang X, Qian W, Yang T, Sun X, Chen Z, Zhu Q: Neuron-derived CCL2 contributes to microglia activation and neurological decline in hepatic encephalopathy. Biol Res 2017, 50:26

33. Starlinger $P$, Haegele S, Wanek D, Zikeli S, Schauer D, Alidzanovic L, Fleischmann E, Gruenberger B, Gruenberger T, Brostjan C: Plasma thrombospondin 1 as a predictor of postoperative liver dysfunction. Br J Surg 2015, 102:826-836

34. Kuroki H, Hayashi H, Nakagawa S, Sakamoto K, Higashi T, Nitta H, Hashimoto D, Chikamoto A, Beppu T, Baba H: Effect of LSKL peptide on thrombospondin 1-mediated transforming growth factor beta signal activation and liver regeneration after hepatectomy in an experimental model. Br J Surg 2015, 102:813-825

35. Jimenez B, Volpert OV, Crawford SE, Febbraio M, Silverstein RL, Bouck N: Signals leading to apoptosis-dependent inhibition of neovascularization by thrombospondin-1. Nat Med 2000, 6:41-48

36. Sipes JM, Krutzsch HC, Lawler J, Roberts DD: Cooperation between thrombospondin-1 type 1 repeat peptides and alpha(v)beta(3) integrin ligands to promote melanoma cell spreading and focal adhesion kinase phosphorylation. J Biol Chem 1999, 274:22755-22762

37. Begany A, Simon M Jr, Dehmel N, Hunyadi J: Expression of thrombospondin-1 (TSP1) and its receptor (CD36) in healthy and diseased human skin. Acta Derm Venereol 1994, 74:269-272

38. Russell WE, Coffey RJ Jr, Ouellette AJ, Moses HL: Type beta transforming growth factor reversibly inhibits the early proliferative response to partial hepatectomy in the rat. Proc Natl Acad Sci U S A $1988,85: 5126-5130$

39. Karkampouna S, Goumans MJ, Ten Dijke P, Dooley S: Kruithof-de Julio M: inhibition of TGFbeta type I receptor activity facilitates liver regeneration upon acute CCl4 intoxication in mice. Arch Toxicol 2016, 90:347-357

40. Bernal W, Hall C, Karvellas CJ, Auzinger G, Sizer E, Wendon J: Arterial ammonia and clinical risk factors for encephalopathy and intracranial hypertension in acute liver failure. Hepatology 2007, 46: $1844-1852$

41. Winterdahl M, Abbas Z, Noer O, Thomsen KL, Gras V, Nahimi A, Vilstrup H, Shah NJ, Dam G: Cerebral water content mapping in cirrhosis patients with and without manifest HE. Metab Brain Dis 2019, 34:1071-1076

42. Rai V, Nath K, Saraswat VA, Purwar A, Rathore RK, Gupta RK: Measurement of cytotoxic and interstitial components of cerebral edema in acute hepatic failure by diffusion tensor imaging. J Magn Reson Imaging 2008, 28:334-341

43. Cheng $\mathrm{C}$, Yu Z, Zhao S, Liao Z, Xing C, Jiang Y, Yang YG, Whalen MJ, Lo EH, Sun X, Wang X: Thrombospondin-1 gene deficiency worsens the neurological outcomes of traumatic brain injury in mice. Int J Med Sci 2017, 14:927-936

44. Koyama Y, Kotani M, Sawamura T, Kuribayashi M, Konishi R, Michinaga S: Different actions of endothelin-1 on chemokine production in rat cultured astrocytes: reduction of CX3CL1/fractalkine and an increase in CCL2/MCP-1 and CXCL1/CINC-1. J Neuroinflammation 2013, 10:51

45. McMillin M, Grant S, Frampton G, Andry S, Brown A, DeMorrow S: Fractalkine suppression during hepatic encephalopathy promotes neuroinflammation in mice. J Neuroinflammation 2016, 13:198

46. Jayakumar AR, Tong XY, Curtis KM, Ruiz-Cordero R, Shamaladevi N, Abuzamel M, Johnstone J, Gaidosh G, Rama Rao KV, Norenberg MD: Decreased astrocytic thrombospondin-1 secretion after chronic ammonia treatment reduces the level of synaptic proteins: in vitro and in vivo studies. J Neurochem 2014, 131: 333-347

47. Bron B, Waldram R, Silk DB, Williams R: Serum, cerebrospinal fluid, and brain levels of bile acids in patients with fulminant hepatic failure. Gut 1977, 18:692-696

48. Jiang W, Desjardins P, Butterworth RF: Cerebral inflammation contributes to encephalopathy and brain edema in acute liver failure: protective effect of minocycline. J Neurochem 2009, 109: 485-493 
49. McMillin M, Frampton G, Quinn M, Ashfaq S, de los Santos M 3rd, Grant S, DeMorrow S: Bile acid signaling is involved in the neurological decline in a murine model of acute liver failure. Am J Pathol 2016, 186:312-323

50. Qureshi MO, Khokhar N, Shafqat F: Ammonia levels and the severity of hepatic encephalopathy. J Coll Physicians Surg Pak 2014, 24: $160-163$

51. Rathi S, Chopra M, Chouduri G, Sharma P, Madan K, Chhabra M, Rai RR, Govil A, Konar A, Goenka M, Agarwal M, Mukherjee J, Thorat V, Salunkhe S, Abraham P, Nagral A, Jhaveri A, Bhat N, Varghese J, RS A, Ravishankar, Reddy DC, Dhiman RK:
Prevalence of minimal hepatic encephalopathy in patients with liver cirrhosis: a cross-sectional, clinicoepidemiological, multicenter, nationwide study in India: the PREDICT Study. J Clin Exp Hepatol 2019, 9:476-483

52. Wang Z, Chu P, Wang W: Combination of rifaximin and lactulose improves clinical efficacy and mortality in patients with hepatic encephalopathy. Drug Des Devel Ther 2019, 13:1-11

53. Oey RC, Buck LEM, Erler NS, van Buuren HR, de Man RA: The efficacy and safety of rifaximin-alpha: a 2-year observational study of overt hepatic encephalopathy. Therap Adv Gastroenterol 2019, 12: 1756284819858256 